This is an electronic reprint of the original article. This reprint may differ from the original in pagination and typographic detail.

Author(s): Kostensalo, Joel; Suhonen, Jouni; Zuber, K.

Title: Shell-model computed cross sections for charged-current scattering of astrophysical neutrinos off $40 \mathrm{Ar}$

Year: $\quad 2018$

Version:

Please cite the original version:

Kostensalo, J., Suhonen, J., \& Zuber, K. (2018). Shell-model computed cross sections for charged-current scattering of astrophysical neutrinos off 40Ar. Physical Review C, 97(3), Article 034309. https://doi.org/10.1103/PhysRevC.97.034309

All material supplied via JYX is protected by copyright and other intellectual property rights, and duplication or sale of all or part of any of the repository collections is not permitted, except that material may be duplicated by you for your research use or educational purposes in electronic or print form. You must obtain permission for any other use. Electronic or print copies may not be offered, whether for sale or otherwise to anyone who is not an authorised user. 


\title{
Shell-model computed cross sections for charged-current scattering of astrophysical neutrinos off ${ }^{40} \mathrm{Ar}$
}

\author{
Joel Kostensalo* and Jouni Suhonen ${ }^{\dagger}$ \\ University of Jyvaskyla, Department of Physics, P.O. Box 35, FI-40014, Finland \\ K. Zuber ${ }^{\ddagger}$ \\ TU Dresden, Institute for Nuclear and Particle Physics, 01069 Dresden, Germany
}

(Received 3 October 2017; revised manuscript received 8 December 2017; published 6 March 2018)

\begin{abstract}
Charged-current (anti)neutrino- ${ }^{40} \mathrm{Ar}$ cross sections for astrophysical neutrinos have been calculated. The initial and final nuclear states were calculated using the nuclear shell model. The folded solar-neutrino scattering cross section was found to be $1.78(23) \times 10^{-42} \mathrm{~cm}^{2}$, which is higher than what the previous papers have reported. The contributions from the $1^{-}$and $2^{-}$multipoles were found to be significant at supernova-neutrino energies, confirming the random-phase approximation (RPA) result of a previous study. The effects of neutrino flavor conversions in dense stellar matter (matter oscillations) were found to enhance the neutrino-scattering cross sections significantly for both the normal and inverted mass hierarchies. For the antineutrino scattering, only a small difference between the nonoscillating and inverted-hierarchy cross sections was found, while the normalhierarchy cross section was 2-3 times larger than that of the nonoscillating cross section, depending on the adopted parametrization of the Fermi-Dirac distribution. This property of the supernova-antineutrino signal could probably be used to distinguish between the two hierarchies in megaton LAr detectors.
\end{abstract}

DOI: 10.1103/PhysRevC.97.034309

\section{INTRODUCTION}

The standard model of particle physics is considered not to be the final theory of the Universe and its constituents. It does not provide a good candidate for dark matter and also the tiny asymmetry between matter and antimatter in the Universe is not understood, not to mention the origin of dark energy. The first point, dark matter, is massively tackled experimentally, as it is expected that such a dark-matter particle will collide with a nucleus, resulting in a measurable recoil energy of a few $\mathrm{keV}$ of the hit nucleus. As is known, cross sections are very small because they have not been observed yet; hence, large detectors with very little radioactive contamination (low background) have to be installed underground to provide the necessary sensitivity. One approach, among others, are large-scale noble gas detectors. One material is argon, which has an abundance of $99.6 \%$ of ${ }^{40} \mathrm{Ar}$ and is currently used in the dark-matter experiments DEAP-3600 [1] and DarkSide-50 [2]. In addition, there are more applications of argon detectors in neutrino physics, one example being the 40-kiloton-scale LArTPC detector envisaged for the planned long-baseline experiment Deep Underground Neutrino Experiment (DUNE) [3]. Here, we would like to focus on cross-sectional determinations on ${ }^{40} \mathrm{Ar}$ for astrophysical neutrinos, which would include solar and supernova neutrinos, neutrinos from the diffuse supernovaneutrino background, low-energy atmospheric neutrinos, or general astrophysical neutrinos with energies below $60 \mathrm{MeV}$.

\footnotetext{
*joel.j.kostensalo@student.jyu.fi

†jouni.suhonen@phys.jyu.fi

${ }^{\ddagger}$ zuber@physik.tu-dresden.de
}

The focus is on $v_{e}$ and $\bar{v}_{e}$ reactions. Starting with the electron neutrinos from the sun, the reaction (1) (see the following section) is possible. Given the fact that the reaction threshold between the mother nucleus ${ }^{40} \mathrm{Ar}$ and the daughter nucleus ${ }^{40} \mathrm{~K}$ is $1.5044 \mathrm{MeV}$, only a fraction of the CNO neutrinos and the ${ }^{8} \mathrm{~B}$ neutrinos can be captured. The neutrino and antineutrino scatterings off ${ }^{40} \mathrm{Ar}$ have previously been studied using the random-phase approximation (RPA) [4,5]. In Ref. [5], the importance of the scattering to the $1^{-}$and $2^{-}$final nuclear states was highlighted. The neutrino scattering ${ }^{40} \operatorname{Ar}\left(\nu, e^{-}\right)^{40} \mathrm{~K}$ has been studied also using the nuclear shell model $[6,7]$ but these investigations have been limited to Gamow-Teller (GT) and Fermi transitions. The shell-model calculations have been complicated by the large number of possible configurations for $s d-p f$ nuclei.

In Ref. [8], a new effective interaction was presented for the $1 s-0 d_{3 / 2}-0 f_{7 / 2}-1 p_{3 / 2}$ model space. This interaction was created to explain the isomeric shift in ${ }^{38} \mathrm{~K}^{\mathrm{m}}$. The model space for which the interaction is designed is significantly smaller than the entire $s d-p f$ model space. This allows for calculations without extra $n \hbar \omega$ type truncations used in the previous shell-model calculations. In the present paper, we have managed to perform shell-model calculations for the neutrino and antineutrino scatterings off ${ }^{40} \mathrm{Ar}$ for multipoles up to $3^{ \pm}$. We give the averaged cross sections folded with both solar- and supernova-neutrino energy spectra, which are of interest from an experimental point of view. In the present paper, we also take into account the effects of neutrino flavor conversions in the dense stellar matter.

This article is organized as follows. In Sec. II, we give the theoretical background behind neutrino-nucleus scattering. In 
Sec. III, we present our results for the total and averaged cross sections, and in Sec. IV, we draw the conclusions.

\section{THEORETICAL FORMALISM}

In this work, we consider charged-current neutrino and antineutrino scattering off the nucleus ${ }^{40} \mathrm{Ar}$, i.e., the reactions

$$
v_{e}+{ }^{40} \operatorname{Ar}\left(0^{+}\right)_{\text {g.s. }} \rightarrow{ }^{40} \mathrm{~K}+e^{-}
$$

and

$$
\bar{\nu}_{e}+{ }^{40} \mathrm{Ar}\left(0^{+}\right)_{\text {g.s. }} \rightarrow{ }^{40} \mathrm{Cl}+e^{+},
$$

where the daughter nucleus can be in an excited state. At the energies considered in this work, the creation of the heavier lepton flavors, $\mu$ and $\tau$, is not energetically possible.

For the solar and core-collapse supernova neutrinos, the four-momentum transfer is small compared to the mass of the exchanged gauge boson $W^{ \pm}$, i.e., $Q^{2}=-q_{\mu} q^{\mu} \ll M_{W^{ \pm}}^{2}$, where the transferred four-momentum is the difference in the momenta of the outgoing and incoming leptons (due to energy conservation also the difference of the momenta of the mother and daughter nuclei is small). Therefore, to a good approximation the scattering can be considered in the lowest order as a single effective vertex with a coupling constant $G=G_{\mathrm{F}} \cos \left(\theta_{\mathrm{C}}\right)$, where $G_{\mathrm{F}}$ is the Fermi constant and $\theta_{\mathrm{C}} \approx 13^{\circ}$ is the Cabibbo angle. The matrix element of this effective Hamiltonian can be written as

$$
\left\langle f\left|H_{\mathrm{eff}}\right| i\right\rangle=\frac{G}{\sqrt{2}} \int d^{3} \mathbf{r} l_{\mu} e^{-\mathbf{q} \cdot \mathbf{r}}\left\langle f\left|\mathcal{J}^{\mu}(\mathbf{r})\right| i\right\rangle,
$$

where $\mathcal{J}^{\mu}$ denotes the hadronic current and $l_{\mu}=$ $e^{\mathbf{q} \cdot \mathbf{r}}\left\langle l\left|j_{\mu}(r)\right| \nu\right\rangle$ [9], where $j_{\mu}$ is the leptonic current.

The initial nuclear state in the scatterings of Eqs. (1) and (2) is the $J_{i}^{\pi_{i}}=0^{+}$ground state of ${ }^{40} \mathrm{Ar}$. We assume that the final nuclear states in ${ }^{40} \mathrm{~K}$ and ${ }^{40} \mathrm{Cl}$ also have well-defined spin parities $J_{f}^{\pi_{f}}$. The double differential cross section for the charged-current (CC) (anti)neutrino-nucleus scattering is given by [9-11]

$$
\begin{aligned}
{\left[\frac{d^{2} \sigma_{i \rightarrow f}}{d \Omega d E_{\mathrm{exc}}}\right]=} & \frac{G^{2}\left|\mathbf{k}_{l^{\mp}}\right| E_{l^{\mp}}}{\pi\left(2 J_{i}+1\right)} F\left( \pm Z_{f}, E_{\mathbf{k}_{l_{\mp}}}\right) \\
& \times\left(\sum_{J \geqslant 0} \sigma_{\mathrm{CL}}^{J}+\sum_{J \geqslant 1} \sigma_{\mathrm{T}}^{J}\right),
\end{aligned}
$$

where $\mathbf{k}_{l^{\mp}}$ and $E_{l^{\mp}}$ are the three-momentum and energy of the outgoing lepton $\left(e^{\mp}\right)$, respectively, and $F\left( \pm Z_{f}, E_{\mathbf{k}_{l \mp}}\right)$ is the Fermi function which accounts roughly for the Coulomb interaction of the final-state lepton and nucleus. The Coulomblongitudinal component $\sigma_{\mathrm{CL}}^{J}$ and the transverse component $\sigma_{\mathrm{T}}^{J}$ in Eq. (4) are defined as

$$
\begin{aligned}
\sigma_{\mathrm{CL}}^{J}= & (1+a \cos \theta)\left|\left(J_{f}\left\|\mathcal{M}_{J}(q)\right\| J_{i}\right)\right|^{2} \\
& +\left(1+a \cos \theta-2 b \sin ^{2} \theta\right)\left|\left(J_{f}\left\|\mathcal{L}_{J}(q)\right\| J_{i}\right)\right|^{2} \\
& +\frac{E_{\mathbf{k}}-E_{\mathbf{k}^{\prime}}}{q}(1+a \cos \theta+c) \\
& \times 2 \operatorname{Re}\left[\left(J_{f}\left\|\mathcal{M}_{J}(q)\right\| J_{i}\right)^{*}\left(J_{f}\left\|\mathcal{L}_{J}(q)\right\| J_{i}\right)\right]
\end{aligned}
$$

and

$$
\begin{aligned}
\sigma_{\mathrm{T}}^{J}= & \left(1-a \cos \theta+b \sin ^{2} \theta\right) \\
& \times\left[\left|\left(J_{f}\left\|\mathcal{T}_{J}^{\mathrm{mag}}(q)\right\| J_{i}\right)\right|^{2}+\left|\left(J_{f}\left\|\mathcal{T}_{J}^{\mathrm{el}}(q)\right\| J_{i}\right)\right|^{2}\right] \\
& \mp \frac{E_{\mathbf{k}}-E_{\mathbf{k}^{\prime}}}{q}(1-a \cos \theta-c) \\
& \times 2 \operatorname{Re}\left[\left(J_{f}\left\|\mathcal{T}_{J}^{\mathrm{mag}}(q)\right\| J_{i}\right)\left(J_{f}\left\|\mathcal{T}_{J}^{\mathrm{el}}(q)\right\| J_{i}\right)^{*}\right] .
\end{aligned}
$$

Here $E_{\mathbf{k}}$ and $E_{\mathbf{k}^{\prime}}$ refer to the energies of the incoming and outgoing leptons. The minus sign is used for the neutrino scattering and the plus sign is for the antineutrino scattering. The quantities $a, b$, and $c$ in Eqs. (5) and (6) are

$$
\begin{aligned}
& a=\sqrt{1-\frac{m_{f}^{2}}{E_{\mathbf{k}^{\prime}}^{2}},} \\
& b=\frac{a^{2} E_{\mathbf{k}} E_{\mathbf{k}^{\prime}}}{q^{2}}, \\
& c=\frac{n_{f}^{2}}{q E_{\mathbf{k}^{\prime}}},
\end{aligned}
$$

where the magnitude of three-momentum transfer $q$ is given by

$$
q=|\mathbf{q}|=\sqrt{\left(E_{\mathbf{k}}-a E_{\mathbf{k}^{\prime}}\right)^{2}+2 a E_{\mathbf{k}} E_{\mathbf{k}^{\prime}}(1-\cos \theta)} .
$$

The final-state interactions are treated in the modified effectivemomentum approximation (MEMA) of Ref. [12]. There the effective momentum inside the nucleus is given by

$$
k_{\text {eff }}=\sqrt{E_{\text {eff }}-m_{e}^{2}},
$$

where $m_{e}$ is the electron mass and $E_{\text {eff }}$ is defined as

$$
E_{\text {eff }}=E_{\mathbf{k}^{\prime}}-V_{\mathrm{C}}(0) \text {. }
$$

The quantity $V_{\mathrm{C}}(0)$ is the value of the Coulomb potential produced by the final-state nucleus at the origin. According to MEMA, we use for small values of $k_{\text {eff }}$ the Fermi function, whereas for the larger values of $k_{\text {eff }}$ we omit the Fermi function in Eq. (4) and replace the energy and three-momentum of the outgoing lepton by the effective values $k_{\text {eff }}$ and $E_{\text {eff }}$.

\section{RESULTS AND DISCUSSION}

\section{A. Nuclear-structure calculations}

The wave functions of the initial and final nuclear states, and the one-body transition densities (OBTDs), related to the transitions, were calculated using the nuclear shell model. The calculations were done using the shell-model code NUSHELLX@MSU [14] in the $1 s-0 d_{3 / 2}-0 f_{7 / 2}-1 p_{3 / 2}$ valence space using the Hamiltonian ZBM2M [8]. In Ref. [8], the proton and neutron excitations across the $N, Z=20$ shell gap were found to be significant in the ${ }^{40} \mathrm{Ca}$ region. Excitations across the shell gap are needed to produce the positive-parity states in ${ }^{40} \mathrm{Cl}$ and ${ }^{40} \mathrm{~K}$ with $J=0,1$, which are the final nuclear states for the Fermi and Gamow-Teller type transitions. The contributions from these multipolarities dominate the (anti)neutrino-scattering cross sections at energies up to few tens of MeVs. In this work, we limit the considered final nuclear 


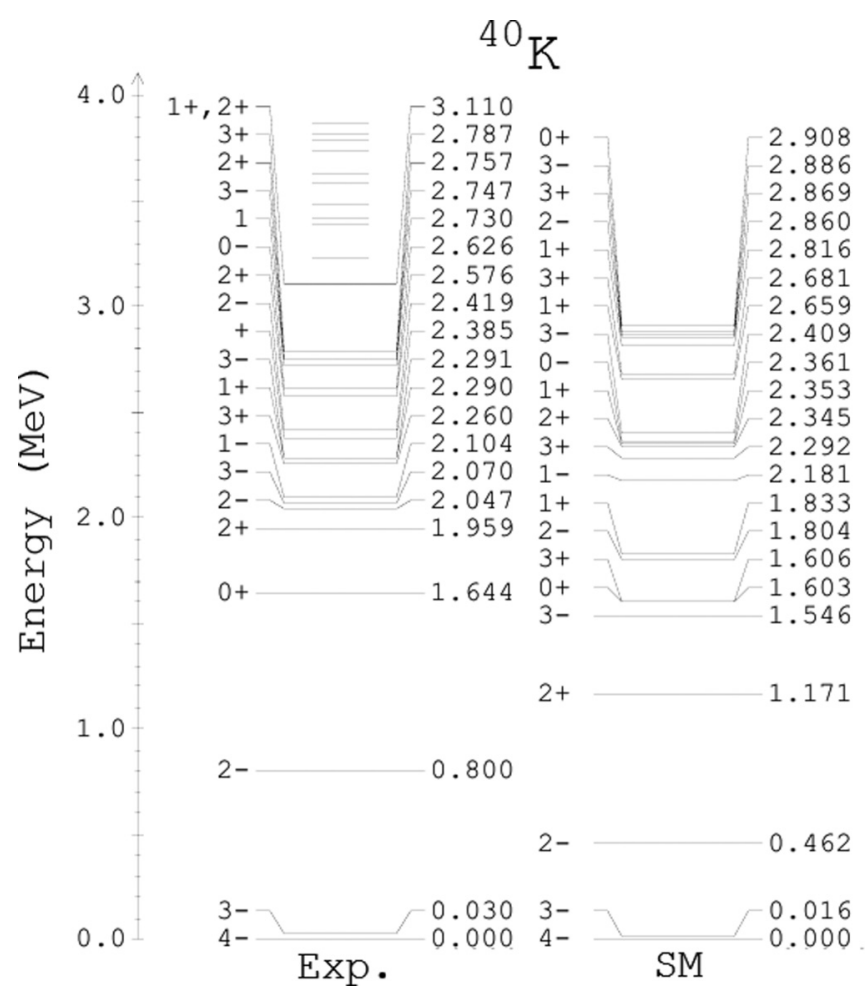

FIG. 1. Experimental and shell-model excitation spectra of ${ }^{40} \mathrm{~K}$. The observed states with uncertain spin parities or angular momenta above 4 are not shown. The experimental spectrum is from Ref. [13].

states to the 100 lowest states for each spin parity up to $J=3$ (800 states). For ${ }^{40} \mathrm{~K}$, this includes states up to $\approx 9 \mathrm{MeV}$ and for ${ }^{40} \mathrm{Cl}$ up to $\approx 7 \mathrm{MeV}$. For the calculation of excited states with higher energies, a larger valence space would be needed. The computational burden of such shell-model calculations is, however, too large for modern computers. In addition, the effective interactions used in shell-model calculations are designed only for low energies. The final nuclear states included here are enough for solar-neutrino energies, but for supernova energies higher excited states can probably contribute. In Ref. [5], the importance of excited states with energies $10-20 \mathrm{MeV}$ for supernova neutrinos was pointed out. The previous randomphase approximation calculations have found the contributions from $0^{+} \rightarrow 4^{ \pm}$scatterings to be very small for energies below $80 \mathrm{MeV}$ [5], and thus the higher multipole contributions can be neglected. As in many previous neutrino-nucleus scattering studies (see, e.g., Refs. $[9,15]$ ), in this work we use the quenched, nuclear-matter value $g_{\mathrm{A}}=1.0$ for the axial-vector coupling constant. This is also consistent with the quenching used in Refs. [6,7]. However, there are indications that for higher neutrino energies the free-nucleon value $g_{\mathrm{A}}=1.27$ might be more appropriate [16]. The uncertainties related to $g_{\mathrm{A}}$ are discussed and estimated in Sec. IIIE.

The experimental and shell-model excitation spectra of ${ }^{40} \mathrm{~K}$ are shown in Fig. 1. The shell model correctly predicts the spin and parity of the ground state and first two excited states. The first $1^{+}$state is predicted to be $450 \mathrm{keV}$ lower than the experimental value. While this increases the contribution of the state, the effect to the averaged cross section is minuscule, since

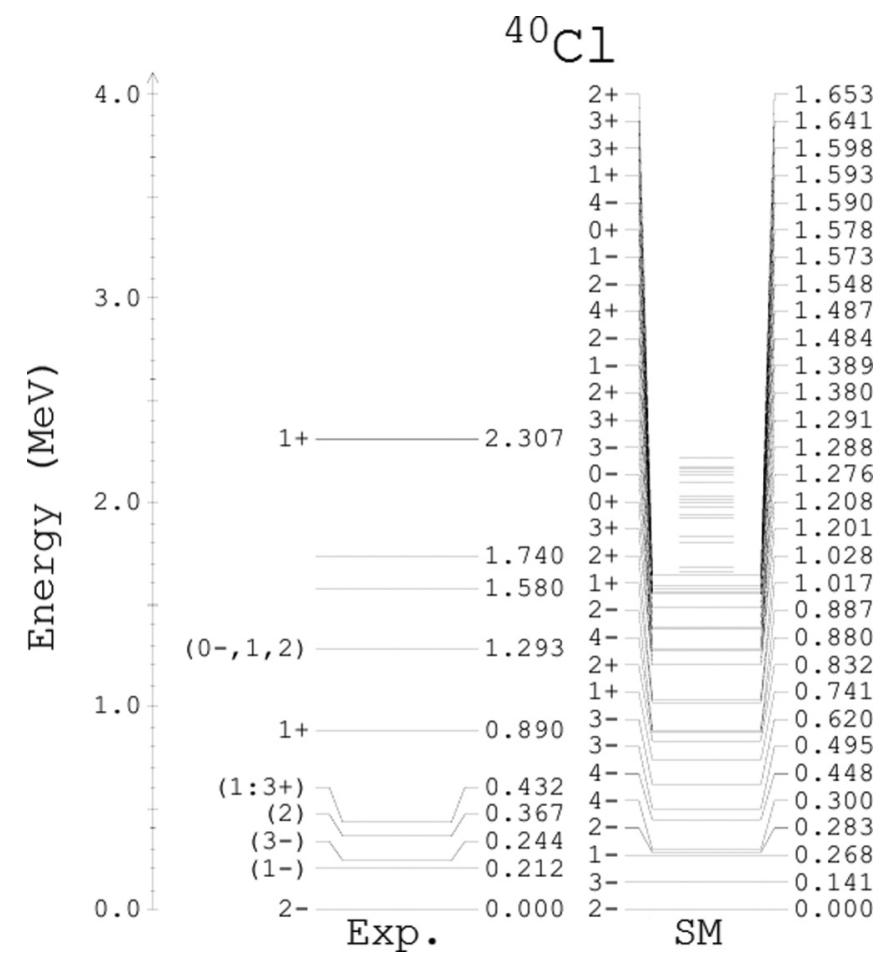

FIG. 2. Experimental and shell-model excitation spectra of ${ }^{40} \mathrm{Cl}$. The observed states with uncertain spin parities or angular momenta above 4 are not shown. The experimental spectrum is from Ref. [13].

the contribution of this state is two magnitudes lower than the dominant contributions (see Fig. 5). The positive-parity state at $2385 \mathrm{keV}$ and the $J=1$ state at $2730 \mathrm{keV}$ are predicted to be $1^{+}$states. For the $\mathrm{O}_{2}{ }^{+}$Isobaric Analog State (IAS) state, the experimental value $4384 \mathrm{keV}$ was adopted.

For ${ }^{40} \mathrm{Cl}$, the excitation spectra are presented in Fig. 2. For this nucleus, the excitation spectrum is not well known experimentally. The shell model is able to predict the spin and parity of the ground state correctly but gets the order of the first $1^{-}$and $3^{-}$states wrong. However, this kind of small deviation from the experimental energies has only small effects on the total folded cross section.

\section{B. Total cross sections}

The total unfolded cross sections as functions of the energy of the incident neutrino for the ${ }^{40} \operatorname{Ar}\left(v, e^{-}\right)^{40} \mathrm{~K}$ scattering and its decomposition to different multipole contributions is shown in Fig. 3 and Table I. The total cross section grows quadratically for energies below $35 \mathrm{MeV}$ and linearly for energies between 35 and $60 \mathrm{MeV}$. Below $25 \mathrm{MeV}$, the total cross section is completely dominated by Gamow-Teller and Fermi transitions. These transitions contribute the most for energies up to $60 \mathrm{MeV}$, but the $2^{-}$transitions become significant for the higher energies. Contributions from other multipoles begin to play a role above $50 \mathrm{MeV}$, but contribute still only a few percent. It is to be expected that the numbers of Table I are realistic up to 40 $\mathrm{MeV}$ (see the discussion in Sec. IIIE2). The RPA calculation of Ref. [5] predicted the scatterings to the $1^{-}$states to contribute approximately as much as the scatterings to the $2^{-}$states for 


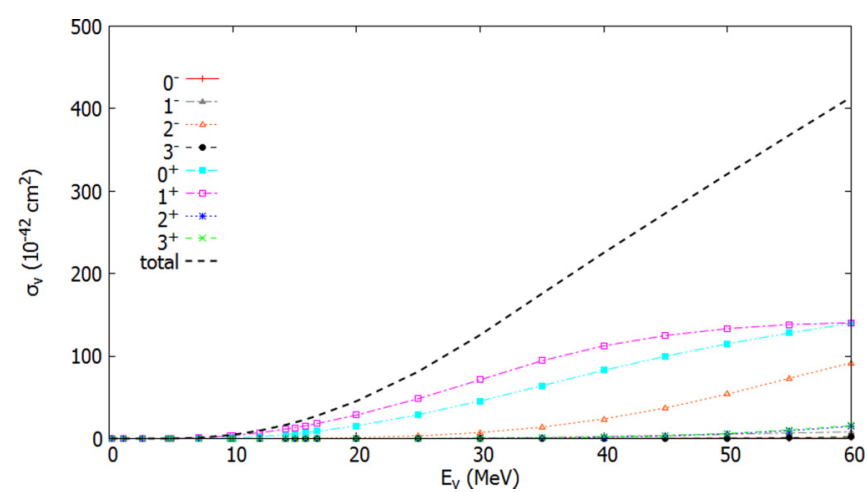

FIG. 3. Unfolded cross sections for the charged-current ${ }^{40} \operatorname{Ar}\left(\nu, e^{-}\right)^{40} \mathrm{~K}$ scattering as functions of the energy of the incident neutrino.

energies below $60 \mathrm{MeV}$. The present shell-model calculation, however, predicts the $1^{-}$contributions to be much lower.

For the antineutrino scattering ${ }^{40} \operatorname{Ar}\left(\bar{v}, e^{-}\right)^{40} \mathrm{Cl}$, the unfolded cross sections are presented in Fig. 4 and Table II as functions of the antineutrino energy. The total cross section grows quadratically for energies up to $60 \mathrm{MeV}$. The total cross section for the antineutrino scattering is only $5 \%$ of the neutrinoscattering cross section for high energies and even less for the lower energies. This is partially explained by the 6-MeV-higher $Q$ value for the $\beta^{-}$decay of ${ }^{40} \mathrm{Cl}$ [17]. The GT transitions dominate for energies below $40 \mathrm{MeV}$ but for higher energies the spin-dipole states $\left(1^{-}\right.$and $\left.2^{-}\right)$are the major contributors. The Fermi transitions do not play a role since there is no isobaric analog state of ${ }^{40} \mathrm{Ar}$ in ${ }^{40} \mathrm{Cl}$.

\section{Averaged solar-neutrino cross sections}

From the experimental point of view, the interesting quantity is the averaged cross section $\langle\sigma\rangle$ which is found by folding the total cross section with the appropriate neutrino energy profile, taking into account the reaction threshold $1.5044 \mathrm{MeV}$. For the solar neutrinos, we adopt a ${ }^{8} \mathrm{~B}$ profile. The differential cross section for the solar-neutrino reaction ${ }^{40} \operatorname{Ar}\left(\nu, e^{-}\right)^{40} \mathrm{~K}$ as a function of excitation energy is presented in Fig. 5(a). The largest contribution in the shell-model calculation comes from the scattering to the third $1^{+}$state at $2659 \mathrm{keV}$. This corresponds to the experimentally observed $J=1$ state at $2730 \mathrm{keV}$. The

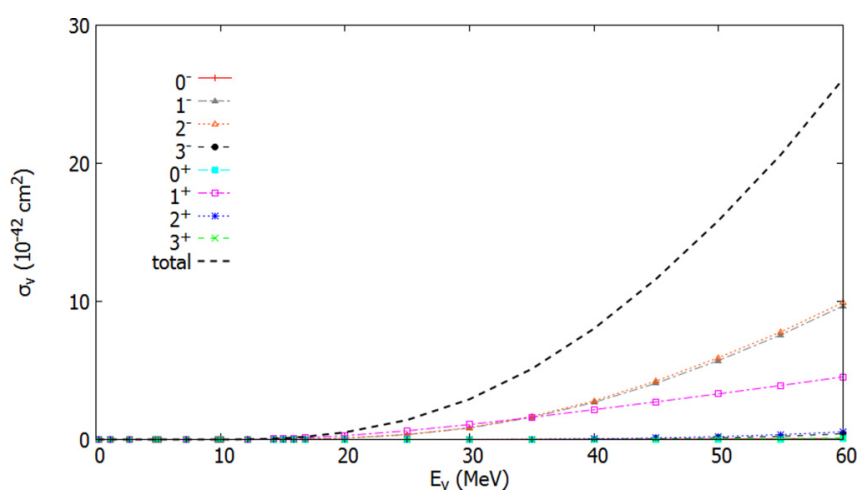

FIG. 4. Unfolded cross sections for the charged-current ${ }^{40} \mathrm{Ar}\left(\bar{\nu}, e^{+}\right){ }^{40} \mathrm{Cl}$ scattering as functions of the energy of the incident neutrino.

transition to the IAS state contributes only $40 \%$ of this. The other significant contributions come from the transition to the $1_{6}{ }^{+}, 1_{10}{ }^{+}, 1_{15}{ }^{+}$, and $1_{17}{ }^{+}$states.

For the solar neutrinos, the total computed cross section is $1.777 \times 10^{-42} \mathrm{~cm}^{2}$, which is higher than the averaged cross sections $1.15(7) \times 10^{-42} \mathrm{~cm}^{2}$ of Ref. [6] and $1.405 \times$ $10^{-42} \mathrm{~cm}^{2}$ of Ref. [7]. The folded cross sections of the previous papers have considered only the GT and IAS transitions. Furthermore, in the previous shell-model calculations, the model space has been chosen as $s d-p f$ with only the $(s d)^{-2}(p f)^{2}$ type of two-particle-two-hole excitations allowed.

\section{Averaged supernova-neutrino cross sections}

\section{Averaged cross sections without neutrino flavor conversions}

The averaged cross section $\langle\sigma\rangle$ for the supernova neutrinos we find by folding the total cross section with a Fermi-Dirac distribution

$$
F_{\mathrm{FD}}\left(E_{\mathbf{k}}\right)=\frac{1}{F_{2}\left(\alpha_{v}\right) T_{v}} \frac{\left(E_{\mathbf{k}} / T_{\nu}\right)^{2}}{1+\exp \left(\mathrm{E}_{\mathbf{k}} / \mathrm{T}_{v}-\alpha_{v}\right)},
$$

where $T_{v}$ is the effective neutrino temperature and $\alpha_{v}$ is the pinching parameter. If the temperature $T_{v}$ and the value of the parameter $\alpha_{v}$ are known, the average neutrino energy is given

TABLE I. Total unfolded cross sections for the neutrino-nucleus scattering ${ }^{40} \operatorname{Ar}\left(v, e^{-}\right)^{40} \mathrm{~K}$ for different neutrino energies, decomposed into the contributing multipolarities. The cross sections are in the units $10^{-42} \mathrm{~cm}^{2}$ and the exponents are shown in parentheses.

\begin{tabular}{|c|c|c|c|c|c|c|c|c|c|}
\hline$E_{v}[\mathrm{MeV}]$ & Total & $0^{+}$ & $0^{-}$ & $1^{+}$ & $1^{-}$ & $2^{+}$ & $2^{-}$ & $3^{+}$ & $3^{-}$ \\
\hline 5.0 & $1.01(-1)$ & $9.27(-9)$ & $3.02(-5)$ & $1.01(-1)$ & $5.56(-7)$ & $1.37(-7)$ & $3.41(-4)$ & $1.51(-7)$ & $8.96(-11)$ \\
\hline 10.0 & $4.56(0)$ & $8.45(-1)$ & $3.25(-3)$ & $3.68(0)$ & $1.55(-3)$ & $9.62(-5)$ & $3.08(-2)$ & $1.19(-4)$ & $1.03(-7)$ \\
\hline 20.0 & $4.68(1)$ & $1.55(1)$ & $5.29(-2)$ & $2.98(1)$ & $1.14(-1)$ & $1.90(-2)$ & $1.27(0)$ & $2.09(-2)$ & $1.36(-4)$ \\
\hline 25.0 & $8.43(1)$ & $2.90(1)$ & $1.01(-1)$ & $5.10(1)$ & $3.79(-1)$ & $8.84(-2)$ & $3.64(0)$ & $9.50(-2)$ & $1.24(-3)$ \\
\hline 30.0 & $1.31(2)$ & $4.55(1)$ & $1.66(-1)$ & $7.55(1)$ & $9.32(-1)$ & $2.95(-1)$ & $8.22(0)$ & $3.12(-1)$ & $6.99(-3)$ \\
\hline 50.0 & $3.42(2)$ & $1.15(2)$ & $5.72(-1)$ & $1.43(2)$ & $7.25(0)$ & $6.46(0)$ & $6.19(1)$ & $6.57(0)$ & $6.19(-1)$ \\
\hline 60.0 & $4.45(2)$ & $1.39(2)$ & $8.50(-1)$ & $1.51(2)$ & $1.13(1)$ & $1.68(1)$ & $1.05(2)$ & $1.67(1)$ & $2.62(0)$ \\
\hline
\end{tabular}


TABLE II. Total unfolded cross sections for the antineutrino-nucleus scattering ${ }^{40} \mathrm{Ar}\left(\bar{\nu}, e^{+}\right)^{40} \mathrm{Cl}$ for different antineutrino energies, decomposed into the contributing multipolarities. The cross sections are in the units $10^{-42} \mathrm{~cm}^{2}$ and the exponents are shown in parentheses.

\begin{tabular}{|c|c|c|c|c|c|c|c|c|c|}
\hline$E_{v}[\mathrm{MeV}]$ & Total & $0^{+}$ & $0^{-}$ & $1^{+}$ & $1^{-}$ & $2^{+}$ & $2^{-}$ & $3^{+}$ & $3^{-}$ \\
\hline \multicolumn{10}{|l|}{5.0} \\
\hline 10.0 & $1.29(-3)$ & $6.58(-12)$ & $2.73(-5)$ & $8.31(-4)$ & $1.96(-6)$ & $1.10(-8)$ & $4.25(-4)$ & $1.01(-10)$ & $2.52(-9)$ \\
\hline 20.0 & $6.10(-1)$ & $1.11(-5)$ & $1.13(-2)$ & $3.76(-1)$ & $1.07(-1)$ & $3.25(-4)$ & $1.15(-1)$ & $9.19(-5)$ & $3.55(-5)$ \\
\hline 25.0 & $1.61(0)$ & $8.94(-5)$ & $2.51(-2)$ & $8.54(-1)$ & $3.58(-1)$ & $2.22(-3)$ & $3.74(-1)$ & $6.12(-4)$ & $3.25(-4)$ \\
\hline 30.0 & $3.24(0)$ & $4.02(-4)$ & $4.44(-2)$ & $1.46(0)$ & $8.48(-1)$ & $9.07(-3)$ & $8.81(-1)$ & $2.43(-3)$ & $1.75(-3)$ \\
\hline 50.0 & $1.67(1)$ & $1.42(-2)$ & $1.76(-1)$ & $4.17(0)$ & $5.87(0)$ & $2.58(-1)$ & $6.06(0)$ & $5.89(-2)$ & $1.17(-1)$ \\
\hline 60.0 & $2.72(1)$ & $4.14(-2)$ & $2.72(-1)$ & $5.56(0)$ & $9.94(0)$ & $6.93(-1)$ & 1.01(1) & $1.41(-1)$ & $4.32(-1)$ \\
\hline
\end{tabular}

by the relation

$$
\left\langle E_{v}\right\rangle / T_{v}=\frac{F_{3}\left(\alpha_{v}\right)}{F_{2}\left(\alpha_{v}\right)},
$$

where

$$
F_{k}\left(\alpha_{\nu}\right)=\int \frac{x^{k} d x}{1+\exp \left(x-\alpha_{v}\right)} .
$$

The normalized differential cross sections for the reaction ${ }^{40} \mathrm{Ar}\left(v, e^{-}\right)^{40} \mathrm{~K}$ as function of the excitation energy are presented in Figs. 5(b) and 5(c). The largest contribution comes from the scattering to the third $1^{+}$state and the transition to the IAS state, both contributing some $50 \%$ of the total. The other significant contributions come from the transition to the $1_{6}{ }^{+}$, $1_{10}{ }^{+}, 1_{15}{ }^{+}$, and $1_{17}{ }^{+}$states, as in the case of the solar neutrinos. The differential cross sections for the reaction ${ }^{40} \mathrm{Ar}\left(\bar{v}, e^{+}\right)^{40} \mathrm{Cl}$ as function of the excitation energy are presented in Fig. 6. The largest contributors are the transitions to $1_{6}{ }^{+}, 2^{-}$g.s. , and $1_{6}{ }^{-}$ states. There are significant contributions coming also from the $22,4^{-}, 1_{4}{ }^{-}$, and $1_{1,2,8,12,19,28}{ }^{+}$states.

In Ref. [4], the ratio of the supernova neutrino and antineutrino cross sections was found to be approximately 12.5 with no flavor conversions included in a scenario with averaged energies $\left\langle E_{v}\right\rangle=11 \mathrm{MeV}$ and $\left\langle E_{\bar{v}}\right\rangle=16 \mathrm{MeV}$ respectively. Folding the cross sections of the present work using FermiDirac distributions with these average energies and assuming $\alpha=0.0$, we find that $\sigma_{\nu}=14.6 \times 10^{-42} \mathrm{~cm}^{2}$ and $\sigma_{\bar{v}}=887 \times$ $10^{-45} \mathrm{~cm}^{2}$ respectively. This gives a ratio of 16.5 for the neutrino and antineutrino cross sections, which is in good agreement with the RPA result of Ref. [4].

\section{Effects of (anti)neutrino flavor conversions}

Because of the large rest masses of both muon and $\tau$ lepton, only electron-flavor (anti)neutrinos are detected in chargedcurrent solar- and supernova-neutrino detection experiments. The energy profile of the (anti)neutrinos detected on Earth is, however, affected by (anti)neutrino flavor conversions (matter oscillations) which take place when the neutrinos propagate through the dense stellar matter [18]. We assume that the muon and $\tau$-neutrino energy profiles are identical, reducing the problem into a two-neutrino one. In the two-neutrino problem, we consider flavor changes $v_{x} \rightarrow v_{e}$, where $v_{x}$ is a linear combination of muon and $\tau$ neutrinos $[19,20]$. The energy profiles of electron neutrinos and antineutrinos reaching Earth can be written as

$$
\begin{aligned}
& F_{v_{e}}\left(E_{\mathbf{k}}\right)=p\left(E_{\mathbf{k}}\right) F_{v_{e}}^{0}\left(E_{\mathbf{k}}\right)+\left[1-p\left(E_{\mathbf{k}}\right)\right] F_{v_{x}}^{0}\left(E_{\mathbf{k}}\right), \\
& F_{\bar{v}_{e}}\left(E_{\mathbf{k}}\right)=\bar{p}\left(E_{\mathbf{k}}\right) F_{\bar{v}_{e}}^{0}\left(E_{\mathbf{k}}\right)+\left[1-\bar{p}\left(E_{\mathbf{k}}\right)\right] F_{\bar{\nu}_{x}}^{0}\left(E_{\mathbf{k}}\right),
\end{aligned}
$$

where $p$ and $\bar{p}$ are the survival probabilities of the electron neutrinos and antineutrinos respectively, and the quantities $F_{v_{e / x}}^{0}\left(E_{\mathbf{k}}\right)$ and $F_{\bar{v}_{e / x}}^{0}\left(E_{\mathbf{k}}\right)$ are the initial energy profiles of the neutrinos and antineutrinos.

For the survival probabilities $p$ and $\bar{p}$, we use the values from Refs. [21,22]. For the normal mass hierarchy, the values are

$$
\begin{aligned}
& p\left(E_{\mathbf{k}}\right)=0, \\
& \bar{p}\left(E_{\mathbf{k}}\right)= \begin{cases}\cos ^{2} \theta_{12}, & E_{\mathbf{k}}<\bar{E}_{s}, \\
0, & E_{\mathbf{k}}>\bar{E}_{s},\end{cases}
\end{aligned}
$$

where $\bar{E}_{s}=18 \mathrm{MeV}$ [22]. For the inverted mass hierarchy, we use the probabilities

$$
\begin{aligned}
& p\left(E_{\mathbf{k}}\right)= \begin{cases}\sin ^{2} \theta_{12}, & E_{\mathbf{k}}<\bar{E}_{s}, \\
0, & E_{\mathbf{k}}>\bar{E}_{s},\end{cases} \\
& \bar{p}\left(E_{\mathbf{k}}\right)=\cos ^{2} \theta_{12},
\end{aligned}
$$

where $\bar{E}_{s}=7 \mathrm{MeV}$ [19]. For the normal hierarchy $\sin ^{2} \theta_{12}=$ 0.306 and for the inverted hierarchy $\sin ^{2} \theta_{12}=0.312$ [23].

For the calculations, we used three sets of parameters $(\alpha$, $T,\langle E\rangle)$ taken from Ref. [24]. The values of the parameters for neutrinos and antineutrinos are presented in Table III.

The folded cross sections for supernova neutrinos are listed in Table IV. The neutrino-scattering cross section is increased roughly three-fold when the neutrino flavor conversions are taken into account. There is little difference between the cross sections with normal and inverted mass hierarchies. For the antineutrino scattering with parameter sets (I) and (II), the inverted-hierarchy cross section is enhanced by $30 \%$ while the normal-hierarchy cross section is enhanced by roughly $100 \%$ relative to the nonoscillating case. For the parameter set (III), the inverted-hierarchy cross section is $67 \%$ larger than the nonoscillating one, while the normal-hierarchy one is almost three times larger than the nonoscillating one. 

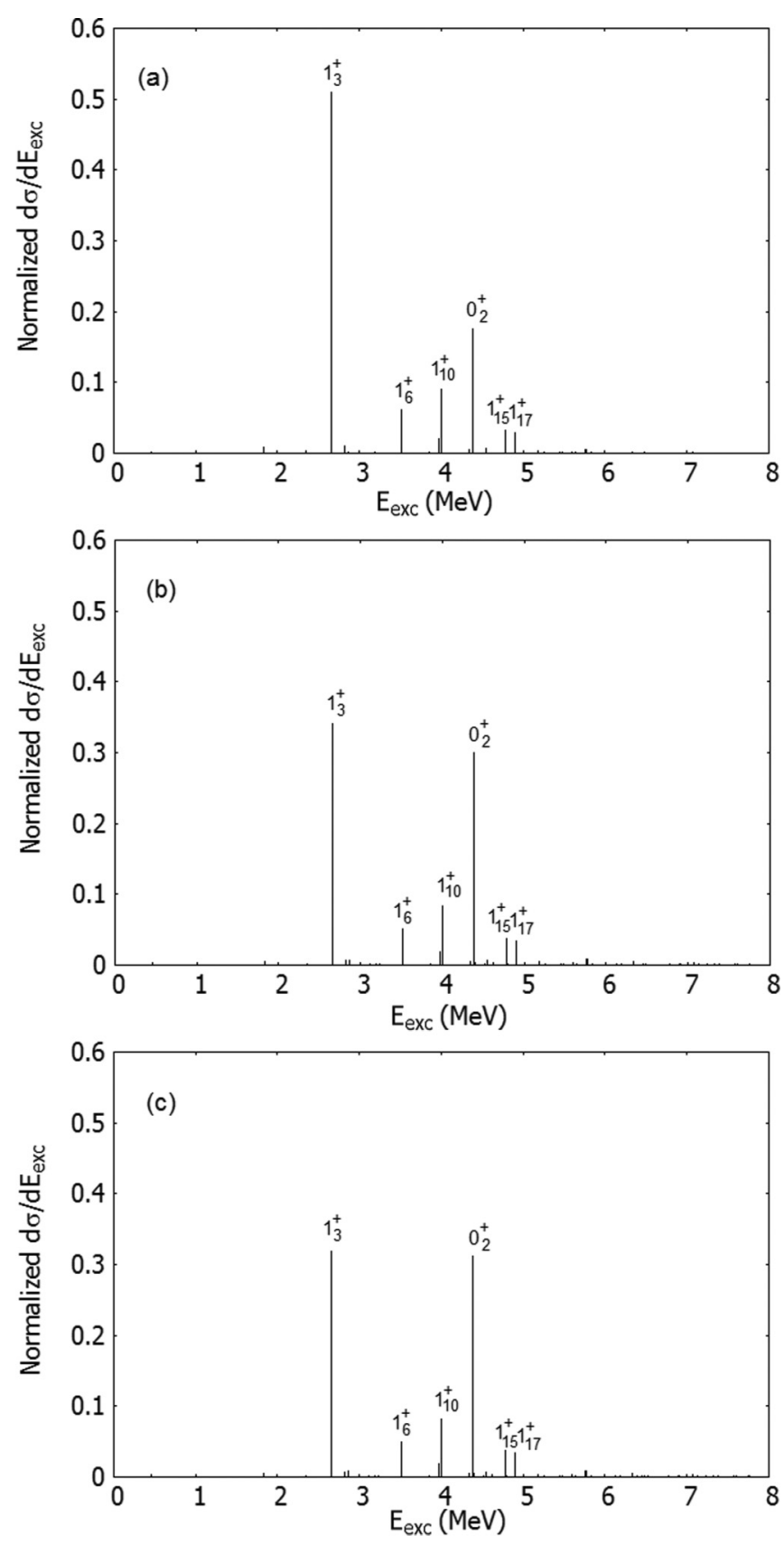

FIG. 5. Differential cross section for the CC supernova-neutrino scattering to final nuclear states at excitation energies $E_{\text {exc }}$. The total cross section is normalized to unity. Panel (a) is folded with the solar neutrino spectrum, panel (b) with Fermi-Dirac distribution with parametrization (I), and panel (c) with parametrization (II) (see Table III). Here the effects of flavor conversions are not included.
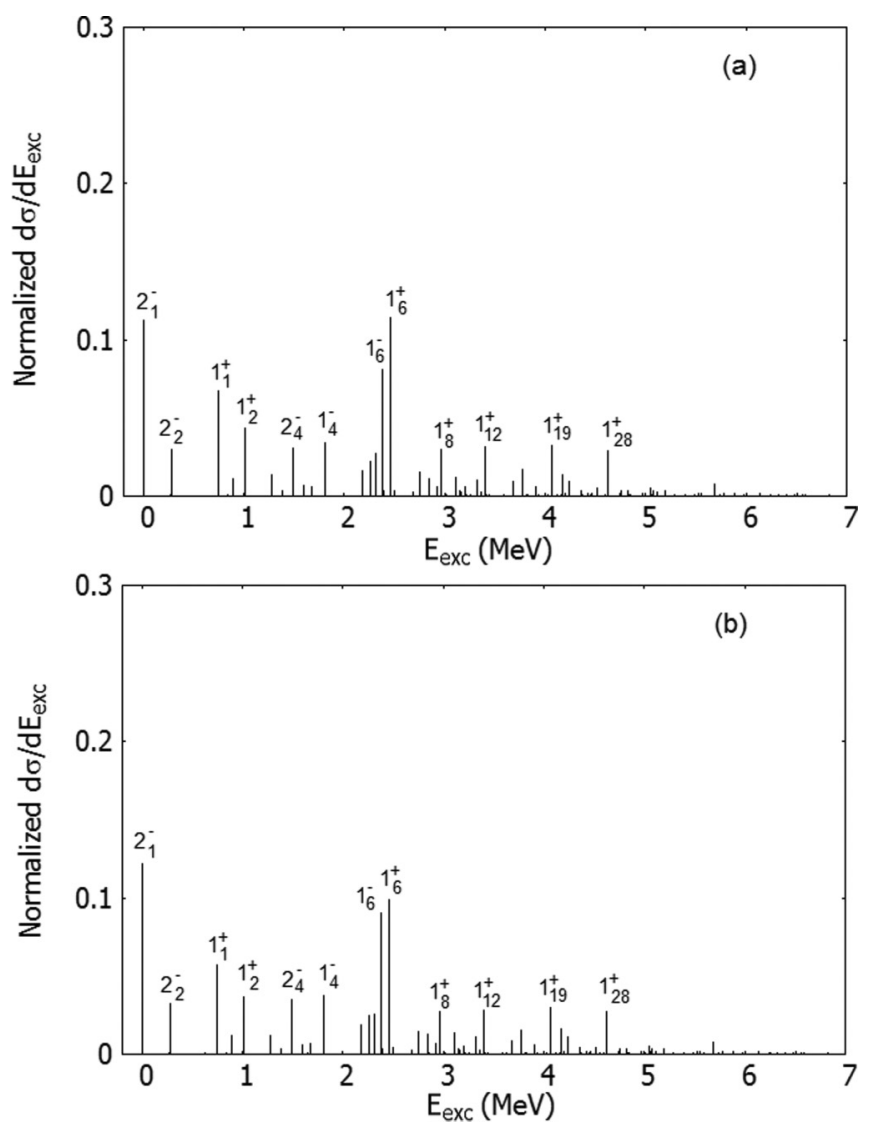

FIG. 6. Differential cross section for the CC supernovaantineutrino scattering to final nuclear states at excitation energies $E_{\text {exc }}$. The total cross section is normalized to unity. Panel (a) with Fermi-Dirac distribution with parametrization (I) and panel (b) with parametrization (II) (see Table III). Here the effects of flavor conversions are not included.

\section{E. Uncertainties}

There are many sources of uncertainties for theoretical estimations of (anti)neutrino-nucleus scattering cross sections, which makes the estimation of the overall uncertainty difficult. In a previous shell-model study [6], the uncertainty was estimated by assuming that each GT strength has a statistical uncertainty of $10 \%$ since generally the uncertainties in the $\mathrm{B}(\mathrm{GT})$ values are of the order 5-10\% [25]. Because of the large number of states in the present study, such statistical uncertainties amount to less than 3\%. For the supernova (anti)neutrinos, there are also uncertainties related to the distribution of neutrino energies, which cannot be accurately estimated. The actual supernova-neutrino spectrum is complex, and the details

TABLE III. Values of parameters $\alpha, T$, and the average neutrino energy $\left\langle E_{v_{e} / x}\right\rangle$ in MeV used for the supernova-neutrino calculations. The values are adopted from Ref. [24]. The index $e$ refers to electron neutrinos and $x=\mu, \tau$ to the nonelectron flavors.

\begin{tabular}{lllll}
\hline \hline & $\left(\alpha_{v_{e}}, T_{v_{e}},\left\langle E_{v_{e}}\right\rangle\right)$ & $\left(\alpha_{\bar{v}_{e}}, T_{\bar{v}_{e}},\left\langle E_{\bar{v}_{e}}\right\rangle\right)$ & $\left(\alpha_{v_{x}}, T_{v_{x}},\left\langle E_{v_{x}}\right\rangle\right)$ & $\left(\alpha_{\bar{v}_{x}}, T_{\bar{v}_{x}},\left\langle E_{\bar{v}_{x}}\right\rangle\right)$ \\
\hline (I) & $(3.0,2.88,11.5)$ & $(3.0,3.41,13.6)$ & $(3.0,4.08,16.3)$ & $(3.0,4.08,16.3)$ \\
(II) & $(0.0,3.65,11.5)$ & $(0.0,4.32,13.6)$ & $(0.0,5.17,16.3)$ & $(0.0,5.17,16.3)$ \\
(III) & $(3.0,2.88,11.5)$ & $(3.0,3.41,13.6)$ & $(0.0,5.17,16.3)$ & $(0.0,5.17,16.3)$ \\
\hline \hline
\end{tabular}


TABLE IV. Folded cross sections in units of $\mathrm{cm}^{2}$ for supernova neutrinos with the quenched value $g_{\mathrm{A}}=1.00$. The first column refers to the parameters of the Fermi-Dirac distribution given in Table III. In the second $\left(v_{e}\right)$ and fifth columns $\left(\bar{v}_{e}\right)$, cross sections for nonoscillating neutrinos are given, while the third, fourth, sixth, and seventh columns give the cross sections with matter oscillations included in the normal mass hierarchy $(\mathrm{NH})$ and in the inverted mass hierarchy $(\mathrm{IH})$.

\begin{tabular}{|c|c|c|c|c|c|c|}
\hline Parameters & $v_{e}$ & $v_{e x}^{\mathrm{NH}}$ & $v_{e x}^{\mathrm{IH}}$ & $\bar{v}_{e}$ & $\bar{v}_{e x}^{\mathrm{NH}}$ & $\bar{v}_{e x}^{\mathrm{IH}}$ \\
\hline (II) & $16.63 \times 10^{-42}$ & $41.70 \times 10^{-42}$ & $42.08 \times 10^{-42}$ & $477.7 \times 10^{-45}$ & $947.3 \times 10^{-45}$ & $624.9 \times 10^{-45}$ \\
\hline
\end{tabular}

of the spectrum are not well known. However, there are two potentially large sources of uncertainty which we can address. The first one is related to the value of the axial-vector coupling constant $g_{\mathrm{A}}$, for which the correct effective value is not well understood. For the $s d$ and $p f$ shells half-life comparisons of GT transitions suggest a quenched value $g_{\mathrm{A}} \approx 1.00$ [26,27]. However, there are indications that for high-energy neutrinos the free-nucleon value $g_{\mathrm{A}}=1.27$ should be used [16]. The effects of $g_{\mathrm{A}}$ are discussed in Sec. IIIE 1. The other potentially large source of systematic uncertainty is related to the limited valence space used in the calculations. Neutrinos with high energies excite states which are out of reach for valence spaces used in the shell-model calculations. The effect of the higher energy states on the folded cross sections are discussed in Sec. IIIE2.

\section{Value of $g_{\mathrm{A}}$}

Adopting the free-nucleon value $g_{\mathrm{A}}=1.27$ instead of the quenched value increases the folded cross section for the solarneutrino-nucleus scattering ${ }^{40} \operatorname{Ar}\left(v, e^{-}\right){ }^{40} \mathrm{~K}$ to $\sigma_{v}=2.642 \times$ $10^{-42} \mathrm{~cm}^{2}$. However, for the solar neutrinos the quenched value $g_{\mathrm{A}}=1.0$ is more appropriate, since the neutrino energies are lower. We assume the uncertainty related to this choice to be $5 \%$ (i.e., $g_{\mathrm{A}}=1.00(5)$ ), which includes most effective values obtained in previous shell-model studies in the $s d$ and $p f$ shells $[26,28]$. The $3 \%$ error related to the nuclear structure can be assumed to be uncorrelated with the error of $g_{\mathrm{A}}$. With these assumptions, we end up with a cross section of $1.78(23) \times$ $10^{-42} \mathrm{~cm}^{2}$ for the solar-neutrino-nucleus scattering.

For the supernova (anti)neutrinos, the results are listed in Table V. Since the energies are several $\mathrm{MeV}$ higher than for the solar neutrinos, the appropriate effective value to be used for $g_{\mathrm{A}}$ is not so clear. It is best to consider the results of Table IV as lower limits and those of Table $\mathrm{V}$ as upper limits for the cross section. Since the GT transitions dominate the folded cross sections, the cross sections scale roughly $\propto g_{\mathrm{A}}^{2}$. Because of this scaling, the ratios between the folded cross sections corresponding to the nonoscillating case, inverted mass hierarchy, and normal mass hierarchy are similar for both the free-nucleon and quenched values of $g_{\mathrm{A}}$.

\section{High-energy neutrinos}

The shell-model computed supernova-neutrino-nucleus cross section for the reaction ${ }^{40} \operatorname{Ar}\left(v, e^{-}\right)^{40} \mathrm{~K}$ is roughly proportional to $E_{v}^{2}$ up to $35 \mathrm{MeV}$, as can be seen from Fig. 7. Beyond that, the shell model predicts roughly a linear energy dependence for the cross section. However, the QRPA result of Ref. [5] predicts larger cross sections and a rough proportionality to $E_{v}^{2}$ for neutrino energies between 40 and $80 \mathrm{MeV}$. Since we expect the QRPA results be more reliable than the shell model ones for higher energies, we need to correct for the missing cross section for the high-energy neutrinos. The small cross sections for high-energy neutrinos stem from the use of a too limited valence space for neutrino scattering at these energies. However, the effects of this on the folded cross sections are expected to be rather modest, since the tail of the supernova-neutrino energy distribution above $35 \mathrm{MeV}$ is quite small and dies out exponentially. The missing strength can be approximated by assuming that the cross section keeps growing proportional to $E_{v}^{2}$ also for energies above $35 \mathrm{MeV}$ in a way which reproduces the QRPA cross sections at the higher energies. In Fig. 7, the total cross section is plotted together with a quadratic fit function which intersects the origin and tangents the shell-model cross section at $35 \mathrm{MeV}$. The resulting fit function agrees with the QRPA results of Ref. [5] at high energies, giving roughly $\sigma=5 \times 10^{-40} \mathrm{~cm}^{2}$ for $60 \mathrm{MeV}$ and $\sigma=8 \times 10^{-40} \mathrm{~cm}^{2}$ for $80 \mathrm{MeV}$. It should be stressed that the energy dependence of the neutrino cross section is not as trivial as $E_{v}^{2}$, but the fitted function can be used to estimate the effect of the missing high-energy states, since it reproduces the 60to $80-\mathrm{MeV}$ QRPA results well, as well as agreeing with the shell-model results for the $35-$ to $40-\mathrm{MeV}$ energies. For the lower energies, the shell-model cross section should of course be used and not that of the fit.

One can estimate the effect of the missing total cross section beyond $40 \mathrm{MeV}$ (this is where the quadratic fit function and the shell-model cross section start to depart from each other) on the folded cross sections. For this, we look at the proportion of neutrinos with energies above $40 \mathrm{MeV}$ in the neutrino-energy profile. The proportion of these neutrinos is the largest for the

TABLE V. Same as Table IV but with the free nucleon value $g_{\mathrm{A}}=1.27$ adopted for the axial-vector coupling constant.

\begin{tabular}{|c|c|c|c|c|c|c|}
\hline Parameters & $v_{e}$ & $v_{e x}^{\mathrm{NH}}$ & $v_{e x}^{\mathrm{IH}}$ & $\bar{v}_{e}$ & $\bar{v}_{e x}^{\mathrm{NH}}$ & $\bar{v}_{e x}^{\mathrm{IH}}$ \\
\hline (II) & $23.03 \times 10^{-42}$ & $56.83 \times 10^{-42}$ & $56.87 \times 10^{-42}$ & $759.1 \times 10^{-45}$ & $1501 \times 10^{-45}$ & $993.2 \times 10^{-45}$ \\
\hline
\end{tabular}




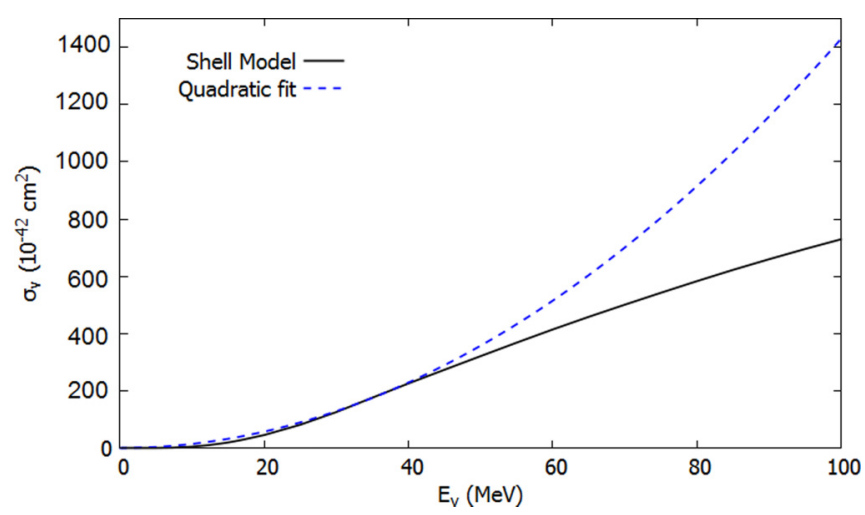

FIG. 7. The total shell-model cross section and a quadratic fit.

parameters $(\alpha=0, T=5.17)$ and it is $1.87 \%$. It then turns out that calculating the folded cross section with the quadratic fit function above $35 \mathrm{MeV}$, instead of the shell-model one, actually increases the folded cross section by about $0.9 \%$. For parameter sets with lower average energies, the effect is significantly reduced from this. For example, for $(\alpha=3, T=2.88)$ the increase is only about $0.01 \%$. Therefore, the lack of computed strength beyond $35 \mathrm{MeV}$ has no practical effect on the folded cross sections calculated in the present paper. However, the numbers of Table I are expected to be realistic only up to $40 \mathrm{MeV}$. As seen in Fig. 4, the roughly quadratic behavior of the total antineutrino cross section continues up to $60 \mathrm{MeV}$ and thus the numbers in Table II should be reliable. Furthermore, one should point out that the most contribution to the folded cross sections, both for the solar as well as for the supernova neutrinos, comes from nuclear excitations at energies that are best described by the nuclear shell model. In this sense, the present results for the folded cross sections should be considered as the best on the market.

\section{CONCLUSIONS}

In the present paper, we have calculated cross sections for the charged-current neutrino and antineutrino scatterings ${ }^{40} \mathrm{Ar}\left(v, e^{-}\right){ }^{40} \mathrm{~K}$ and ${ }^{40} \mathrm{Ar}\left(\bar{v}, e^{+}\right){ }^{40} \mathrm{Cl}$ for astrophysical neutrinos with energies below $60 \mathrm{MeV}$, especially relevant for both solar and supernova neutrinos. The initial and final nuclear wave functions were calculated using the nuclear shell model. The effects of (anti)neutrino flavor conversions in dense stellar matter (matter oscillations) were also included.

While the previous shell-model calculations of cross sections have considered only Gamow-Teller and Fermi types of transitions, in the present paper we consider also transitions to the first $1000^{ \pm}, 1^{ \pm}, 2^{ \pm}$, and $3^{ \pm}$states. Contributions from the spin-dipole states $\left(1^{-}\right.$and $\left.2^{-}\right)$dominate the total unfolded antineutrino-scattering cross section for antineutrino energies above $40 \mathrm{MeV}$. Especially the lowest two $2^{-}$states and the $6^{\text {th }} 1^{-}$state give significant contributions to the cross section. For the total unfolded neutrino cross section, the transitions to $2^{-}$states in ${ }^{40} \mathrm{~K}$ becomes significant above $30 \mathrm{MeV}$ of neutrino energy. It is therefore clear that the $1^{-}$and $2^{-}$multipolarities should not be omitted in the cross-sectional calculations.

For solar neutrinos, with a ${ }^{8} \mathrm{~B}$ distribution, the folded cross section was found to be $\sigma_{v}=1.78(23) \times 10^{-42} \mathrm{~cm}^{2}$. This cross section is higher than the folded cross sections $1.15(7) \times 10^{-42} \mathrm{~cm}^{2}$ [6] and $1.405 \times 10^{-42} \mathrm{~cm}^{2}$ [7] of previous theoretical papers, where only Fermi and Gamow-Teller transitions were taken into account.

We also computed the folded scattering cross sections for supernova (anti)neutrinos. To test the scope of our calculations, the cross sections were also folded with the supernova-neutrino spectra used in the RPA-based calculation of Ref. [4] with no matter oscillations included. The neutrino-antineutrino crosssectional ratio was found to be 16.5 , which is in a reasonable agreement with the ratio 12.5 reported in Ref. [4]. Though the limited valence space considered in the present paper is unable to account for giant resonances, the systematic uncertainty related to this propagates to the folded cross sections at the level of $1 \%$ or less, as verified by our analysis of the previous RPA results. Since the folded cross sections for both the solar and supernova neutrinos gather the relevant contributions from nuclear excitations best described by the nuclear shell model, the present results for the folded cross sections can be considered as the most accurate ones available thus far.

For supernova-neutrino scattering, the cross section is increased roughly threefold when the neutrino flavor conversions are taken into account. The results for normal and inverted mass hierarchies agreed with $1 \%$ accuracy for all three adopted Fermi-Dirac distribution parameter sets (see Table III). For parametrizations (I) and (II), the antineutrino-scattering cross sections in the inverted-hierarchy scenario were enhanced by $30-35 \%$ with respect to the nonoscillating ones, while for the parameter set (III) the inverted-hierarchy cross section was $67 \%$ larger. The normal-hierarchy cross section was roughly twice as large as the nonoscillating cross section for the first two parameter sets and three times larger for the parameter set (III). One can then speculate that the strong effects of the neutrino mass hierarchy on the supernova-antineutrino signal could be used to distinguish between the two hierarchies in megaton LAr detectors. On the other hand, if the hierarchy was known, one could learn about the flavor-conversion processes inside the supernovae.

\section{ACKNOWLEDGMENT}

This work has been partially supported by the Academy of Finland (Suomen Akatemia) under the Finnish Centre of Excellence Programme 2012-2017 (Nuclear and Accelerator Based Programme at JYFL).
[1] P.-A. Amaudruz, M. Baldwin, M. Batygov, B. Beltran, C. E. Bina, D. Bishop, J. Bonatt, G. Boorman, M. G. Boulay, B. Broerman et al., arXiv:1707.08042.

[2] P. Agnes et al., Phys. Lett. B 743, 456 (2015).
[3] R. Acciarri, M. A. Acero, M. Adamowski, C. Adams, P. Adamson, S. Adhikari, Z. Ahmad, C. H. Albright, T. Alion, E. Amador et al., arXiv.1512.06148. 
[4] I. Gil-Botella and A. Rubbia, J. Cosmol. Astropart. Phys. 2003, 009 (2003).

[5] M.-K. Cheoun, E. Ha, and T. Kajino, Phys. Rev. C 83, 028801 (2011).

[6] W. E. Ormand, P. M. Pizzochero, P. F. Bortignon, and R. A. Broglia, Phys. Lett. B 345, 343 (1995).

[7] T. Suzuki and M. Honma, Phys. Rev. C 87, 014607 (2013).

[8] M. L. Bissell, J. Papuga, H. Naïdja, K. Kreim, K. Blaum, M. De Rydt, R. F. Garcia Ruiz, H. Heylen, M. Kowalska, R. Neugart, G. Neyens, W. Nörtershäuser, F. Nowacki, M. M. Rajabali, R. Sanchez, K. Sieja, and D. T. Yordanov, Phys. Rev. Lett. 113, 052502 (2014).

[9] E. Ydrefors and J. Suhonen, Adv. High Energy Phys. 2012, 373946 (2012).

[10] E. Kolbe, K. Langanke, G. Martínez-Pinedo, and P. Vogel, J. Phys. G: Nucl. Part. Phys. 29, 2569 (2003).

[11] E. Ydrefors and J. Suhonen, Phys. Rev. C 87, 034314 (2013).

[12] J. Engel, Phys. Rev. C 57, 2004 (1998).

[13] National Nuclear Data Center, Brookhaven National Laboratory [www.nndc.bnl.gov]

[14] B. A. Brown and W. D. M. Rae, Nucl. Data Sheets 120, 115 (2014).

[15] W. Almosly, B. G. Carlsson, J. Suhonen, J. Toivanen, and E. Ydrefors, Phys. Rev. C 94, 044614 (2016).
[16] T. Katori and M. Martini, J. Phys. G: Nucl. Part. Phys. 45, 013001 (2018).

[17] J. Chen, Nucl. Data Sheets 140, 1 (2017).

[18] B. Dasgupta, A. Dighe, G. G. Raffelt, and A. Y. Smirnov, Phys. Rev. Lett. 103, 051105 (2009).

[19] J. Gava and C. Volpe, Phys. Rev. D 78, 083007 (2008).

[20] A. B. Balantekin and G. M. Fuller, Phys. Lett. B 471, 195 (1999).

[21] G. G. Raffelt, Prog. Part. Nucl. Phys. 64, 393 (2010).

[22] G. Martínez-Pinedo, B. Ziebarth, T. Fischer, and K. Langanke, Eur. Phys. J. A 47, 98 (2010).

[23] K. Nakamura et al. (Particle Data Group), J. Phys. G: Nucl. Part. Phys. 37, 075021 (2010).

[24] M. T. Keil, G. G. Raffelt, and H.-T. Janka, Astrophys. J. 590, 971 (2003).

[25] B. A. Brown and B. H. Wildenthal, At. Data Nucl. Data Tables 33, 347 (1985).

[26] B. H. Wildenthal, M. S. Curtin, and B. A. Brown, Phys. Rev. C 28, 1343 (1983).

[27] G. Martínez-Pinedo, A. Poves, E. Caurier, and A. P. Zuker, Phys. Rev. C 53, R2602(R) (1996).

[28] T. Siiskonen, M. Hjorth-Jensen, and J. Suhonen, Phys. Rev. C 63, 055501 (2001). 